\title{
Assessment of dermal papillary and microvascular parameters in psoriasis vulgaris using in vivo reflectance confocal microscopy
}

\author{
ALEXANDRA BATANI $^{1 *}$, DACIANA ELENA BRĂNISTTEANU ${ }^{2 *}$, MIHAELA ADRIANA ILIE $^{1 *}$, DANIEL BODA $^{1 *}$, \\ SIMONA IANOSI $^{3 *}$, GABRIEL IANOSI $^{4 *}$ and CONSTANTIN CARUNTU ${ }^{5,6^{*}}$ \\ ${ }^{1}$ Dermatology Research Laboratory, Carol Davila University of Medicine and Pharmacy, 050474 Bucharest; \\ ${ }^{2}$ Department of Dermatology, 'Grigore T. Popa' University of Medicine and Pharmacy, 700115 Iassy; ${ }^{3}$ Department of \\ Dermatology, University of Medicine and Pharmacy; ${ }^{4}$ Department of Surgery, Clinical Military Hospital, University of \\ Medicine and Pharmacy, 200349 Craiova; ${ }^{5}$ Physiology Department, Carol Davila University of Medicine and Pharmacy, \\ 050474 Bucharest; ${ }^{6}$ Department of Dermatology, N. Paulescu National Institute of Diabetes, \\ Nutrition and Metabolic Diseases, 011233 Bucharest, Romania
}

Received May 1,2017; Accepted September 6, 2017

DOI: $10.3892 /$ etm.2017.5542

\begin{abstract}
In vivo reflectance confocal microscopy (RCM) is a modern, non-invasive imaging technique, which allows for real-time examination of the upper layers of the skin at a resolution similar to that of classic microscopy. In addition, it has the advantage of real-time evaluation of blood flow and dynamic monitoring of cutaneous changes while preserving tissue integrity. The present study reported on the in vivo RCM technique as an objective method for the noninvasive assessment of psoriasis vulgaris that is potentially applicable in clinical studies and in monitoring the evolution of lesions under treatment. In psoriasis lesions, RCM virtual horizontal sections at the level of the dermo-epidermal junction featured numerous and prominent dermal papillae that were not surrounded by bright rings of basal cells. Micromorphological examination of the lesions using this technique revealed that mean values of the section area, the perimeter and the Feret's diameter of the dermal papillae were significantly higher in psoriatic lesions than in normal skin. An increased number of capillary vessels per lesional dermal papilla as compared to healthy skin was observed. Furthermore, micromorphological parameters of dermal capillaries were increased
\end{abstract}

Correspondence to: Dr Daciana Elena Brănișteanu, Department of Dermatology, 'Grigore T. Popa' University of Medicine and Pharmacy, 16 Universitatii Street, 700115 Iassy, Romania

E-mail: debranisteanu@yahoo.com

Dr Simona Ianosi, Department of Dermatology, University of Medicine and Pharmacy, 2-4 Petru Rares Street, 200349 Craiova, Romania

E-mail: simonaianosi@hotmail.com

${ }^{*}$ Contributed equally

Key words: reflectance confocal microscopy, psoriasis vulgaris in psoriatic skin. These observations point to the utility of in vivo RCM as a promising technique for the non-invasive diagnosis of psoriasis vulgaris, for monitoring the evolution of lesions at a micromorphological level under various treatments and for gaining a better understanding of the pathophysiological processes that occur in the evolution of this disease.

\section{Introduction}

Psoriasis is a worldwide recurrent inflammatory skin disorder with a high prevalence in the general population $(1,2)$ and a major negative impact on the quality of life of affected patients (3). The pathophysiological mechanisms involved in psoriasis are complex, including genetic predisposition, the influence of immunologic factors and environmental triggers $(4,5)$. Effective treatment of psoriasis is often challenging $(6,7)$. Despite its characteristic clinical aspects, histological examination remains the gold standard in psoriasis diagnosis $(8,9)$. However, skin biopsy is invasive and may be associated with various complications, including infection and scaring. Histological examination only provides information on one specific tissue area and on the status of the tissue at the time it is excised. In addition, in early stages of the disease, the histopathological changes may be modest and the results are inconclusive. Skin biopsy is not useful for dynamic monitoring of skin changes during treatment, as it is not possible to evaluate the same area over time (9-11).

In vivo reflectance confocal microscopy (RCM) is a novel imaging tehnique that offers noninvasive, real-time visualization of the superficial layers of the skin down to the papillary dermis, with a resolution that allows for examination of cellular details. The images are obtained and processed rapidly and in real-time, and the area may be reviewed whenever necessary to assess the dynamic changes of the cutaneous tissue during various pathophysiological processes or the response to treatment. In addition, in contrast to conventional skin histology images, RCM optical sections are in the horizontal plane, 
enabling a detailed examination of a wide skin area at a given depth (10-18).

In the present study, in vivo RCM was used for the objective assessment of dermal papillary and microvascular parameters in skin lesions of psoriasis vulgaris patients. RCM is a method potentially applicable in clinical studies for monitoring the evolution of the lesions under treatment, which may be useful for gaining a better understanding of the pathophysiological processes involved in this disease and to determine noninvasive diagnostic criteria that may replace classic histopathology.

\section{Materials and methods}

Patients. The present study was performed at the Department of Dermatology, Nicolae Paulescu National Institute of Diabetes, Nutrition and Metabolic Disease (Bucharest, Romania) in accordance with the guidelines of the local ethics committee. A total of 23 subjects (12 male and 11 female; $35.22 \pm 2.736$ years old) were enrolled in the study, written informed consent was obtained from all participants prior to their inclusion in the study.

Of these patients, 9 were treated for plaque psoriasis. The median age of this group of patients was $38.44 \pm 4.664$ years, and the male-to-female ratio was 5:4. The lesions were characteristic erythematosquamous plaques and were located on the trunk and limbs. The diagnosis was established by clinical observation and further confirmed by histological examination. None of the patients was under systemic or topical treatment at the time of the first clinical examination.

The other 14 subjects were healthy volunteers who were age- and gender-matched with the Psoriasis group. In this group, there was an even number of males and females and the median age was $33.14 \pm 3.371$ years.

Study protocol. Patients were examined under medical supervision, under standard temperature $\left(22 \pm 1^{\circ} \mathrm{C}\right)$ and humidity $(50 \pm 5 \%)$, and in a comfortable position. On the area of the skin to be analyzed, cleaned and dried in advance, a few drops of immersion oil (crodamol) were applied to engage the optical disc and reduce refractive irregularities.

In the Psoriasis group, a clinically significant lesion that was easily accesible for examination was selected for each subject. Lesions covered by thick scales or located in hairy areas were avoided. In the Control group, the RCM images were taken from the healthy skin of the dorsal non-dominant hand.

RCM images were obtained using a VivaScope 1500 confocal microscope (Lucid Inc., Rochester, NY, USA) with a $785 \mathrm{~nm}$ wavelength and a lighting power below $30 \mathrm{~mW}$.

The RCM images were examined using Image $\mathbf{J} 1.45$ image analysis software (National Institutes of Health, Bethesda, MD, USA). The percentage of dermal tissue area from the total examined cutaneous tissue area at the dermo-epidermal junction was determined by evaluating 8 horizontal 500x500 $\mu \mathrm{m}$ sections for each subject; the areas of all identifiable dermal papillae were measured. Furthermore, the micromorphological parameters of dermal papillae and dermal capillaries were assessed. Parameters of interest were area, perimeter and Feret's diameter (Fig. 1). For each patient, a minimum number
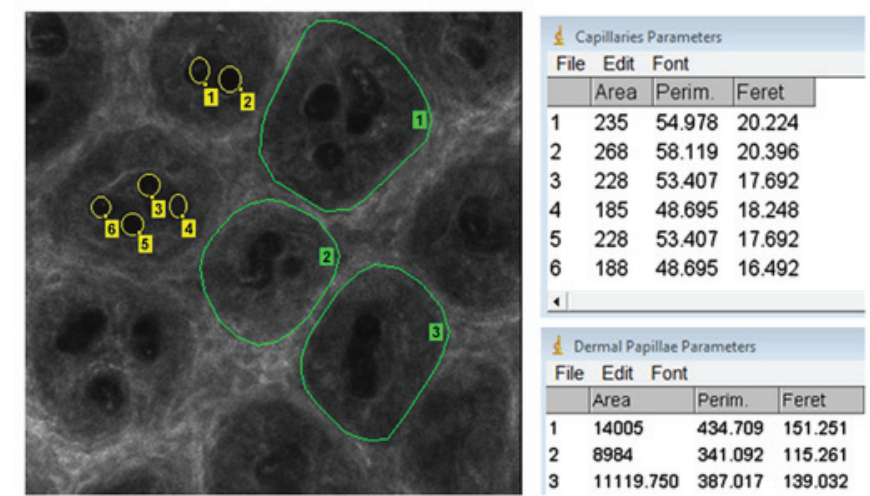

Figure 1. Micromorphological parameters of dermal papillae and dermal capillaries. The parameters of the dermal papillae (green contour lines) and of capillary vessels (yellow circles) were measured using Image J 1.45 analysis software. The parameters investigated were area, Perim and Feret. Perim, perimeter; Feret, Feret's diameter.

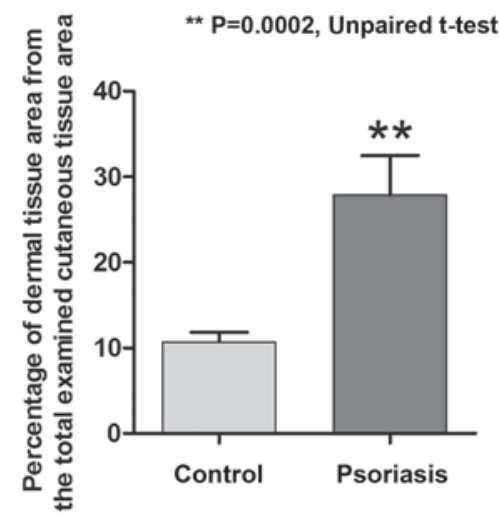

Figure 2. Difference between the percentage of dermal tissue from the total examined cutaneous tissue at the level of the dermo-epidermal junction. Due to the papillomatosis present in the lesional skin, a larger area of dermal tissue was also identified on the reflectance confocal microscopy images. Values are expressed as the mean \pm standard deviation. ${ }^{* *} \mathrm{P}=0.0002$.

of 30 dermal papillae and 30 dermal capillaries was examined and measured.

Statistical analysis. Statistical analysis of the data was performed using GraphPad Prism 6 software (GraphPad Inc., La Jolla, CA, USA). The unpaired t-test was used to determine the statistical significance of differences between groups. $\mathrm{P}<0.05$ was considered to indicate a statistically significant difference. Values are expressed as the mean \pm standard error of the mean.

\section{Results}

Assessment of dermal papillae of psoriasis patients by RCM. In psoriasis lesions, RCM virtual horizontal sections at the level of the dermo-epidermal junction exibited numerous and prominent dermal papillae, with polygonal shapes, separated by thin epidermal ridges; they were well defined, but unlike dermal papillae of healthy skin, they were not surrounded by any bright ring of basal cells. In psoriasis lesions, the area of papillary dermal tissue accounted for $27.90 \pm 4.577 \%$ of the total examined cutaneous tissue area, which was significantly 


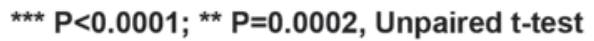
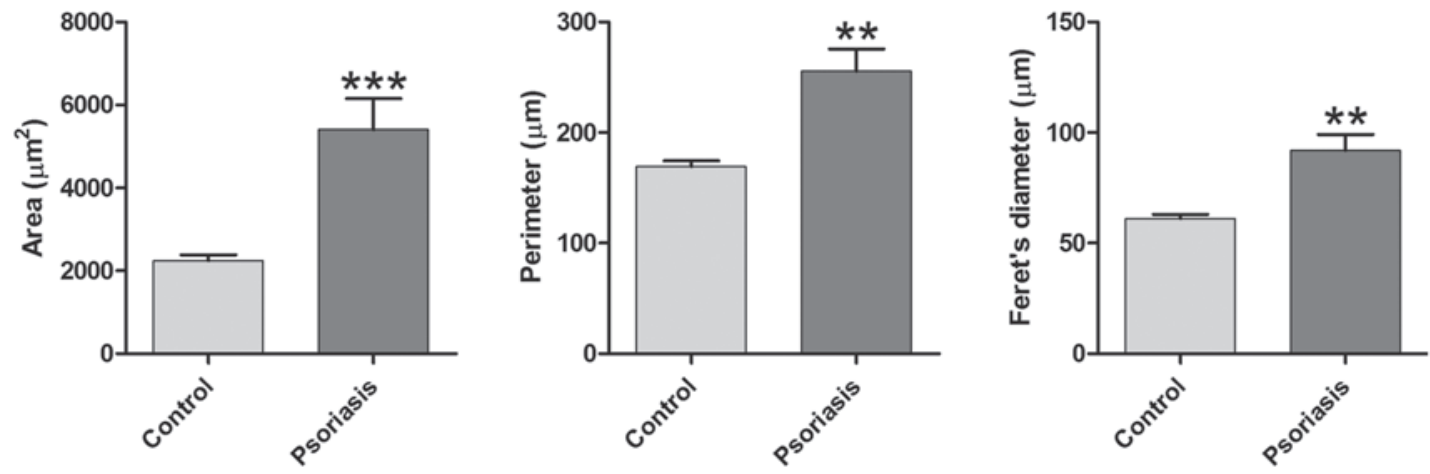

Figure 3. Comparison of parameters of dermal papillae (area, perimeter, Feret's diameter) between the two study groups. All three parameters were significantly increased in the skin affected by psoriasis, as compared with those in healthy skin. Values are expressed as the mean \pm standard deviation. ${ }^{* * * *} \mathrm{P}<0.0001$, ${ }^{* *} \mathrm{P}=0.0002$.
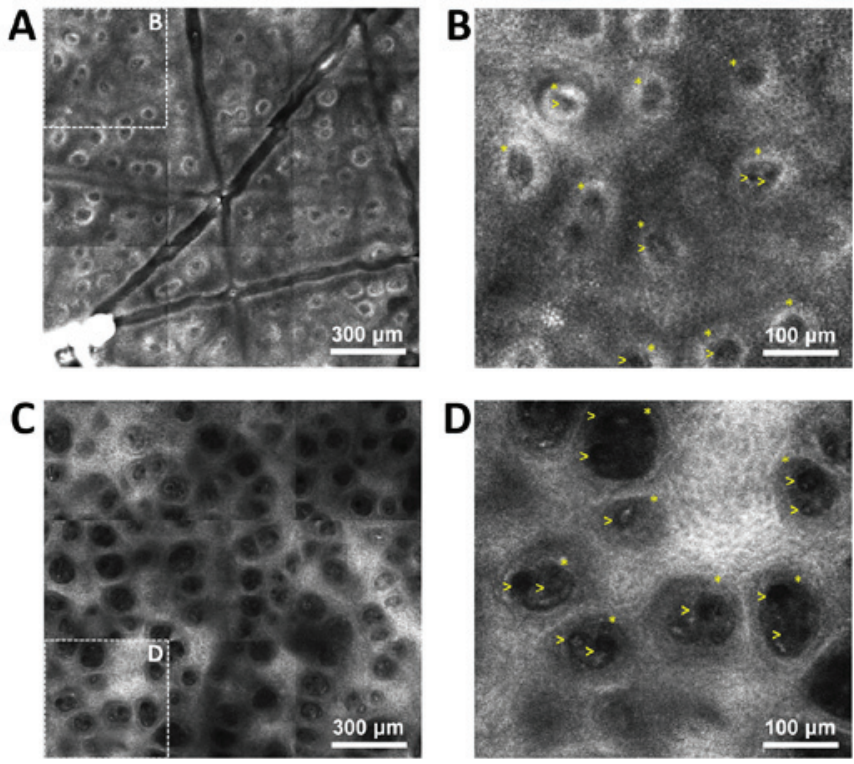

Figure 4. RCM images from psoriasis lesions and healthy skin. (A) RCM mosaic of $1.5 \times 1.5 \mathrm{~mm}$ from the skin of a healthy volunteer; dermal papillae are visible in the dermo-epidermal junction as round-oval, dark areas with homogeneous structure, surrounded by bright rings of basal cells. (B) Magnified window from (A), in which capillaries are visible as round black structures (yellow arrowheads) in the center of dermal papillae (yellow asterisks). (C) RCM mosaic of $1.5 \times 1.5 \mathrm{~mm}$ at the dermo-epidermal junction from the skin of a psoriasis patient. The image reveals increased size of the dermal papillae, which are separated by a thin epithelial septum, while papillary rings are absent. (D) Magnified window from $C$ exhibiting enlarged papillae and basal cells (yellow asterisks) filled with prominent, round and dark structures that are indicative of dilated vessels (yellow arrows), while the bright rings around them in B are absent (scale bar, 300 or $100 \mu \mathrm{m})$. RCM, reflectance confocal microscopy.

higher than the area determined for healthy skin $(10.70 \pm 1.163 \%$; $\mathrm{P}=0.0002$; Fig. 2). Micromorphological examination using the RCM technique revealed that the mean values of the section area, the perimeter and the Feret's diameter of the dermal papillae were significantly higher in psoriatic lesions than in normal skin. The mean value of the section area of dermal papillae was significantly increased to $5405 \pm 749.0 \mu \mathrm{m}$ in the Psoriasis group as compared with $2243 \pm 139.0 \mu \mathrm{m}$ in the Control group $(\mathrm{P}<0.0001)$. In addition, a significant difference

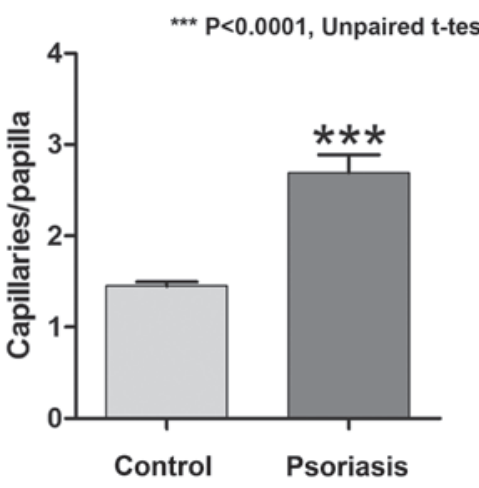

Figure 5. Difference between skin affected by psoriasis and healthy skin regarding the mean number of capillary sections in each dermal papillae. Values are expressed as the mean \pm standard deviation. ${ }^{* * *} \mathrm{P}<0.0001$.

between the two groups was identified regarding the papillae's perimeter, with a mean value of $255.6 \pm 20.02 \mu \mathrm{m}$ in the skin affected by psoriasis compared with $169.4 \pm 5.127 \mu \mathrm{m}$ in the skin of the healthy volunteers $(\mathrm{P}=0.0002)$. The Feret's diameter of the dermal papillae in the Psoriasis group was also increased to $91.94 \pm 7.201 \mu \mathrm{m}$ compared with $61.03 \pm 1.913 \mu \mathrm{m}$ in the Control group ( $\mathrm{P}=0.0002)$. Fig. 3 summarizes the differences in the parameters of dermal papillae evaluated by RCM between the two groups of patients.

Assessment of dermal capillaries of psoriasis patients by $R C M$. Dermal capillaries may be visualized on RCM virtual sections as dark, round structures in the center of dermal papillae and blood flow may be observed via real-time imaging. The pathophysiology of psoriasis vulgaris includes changes of the capillary vessels to become dilated and tortous, describing multiple loops in their trajectory, which was successfully identified in the RCM images (Fig. 4). An increased number of capillary sections per lesional dermal papilla was present as compared with that in healthy skin, with $2.691 \pm 0.199$ capillary sections per papilla in the psoriasis group as compared with $1.451 \pm 0.0447$ in the control group; the difference was statistically significant $(\mathrm{P}<0.0001$; Fig. 5). The micromorphological parameters of dermal capillaries were also increased in psoriatic skin. Regarding the section area of 

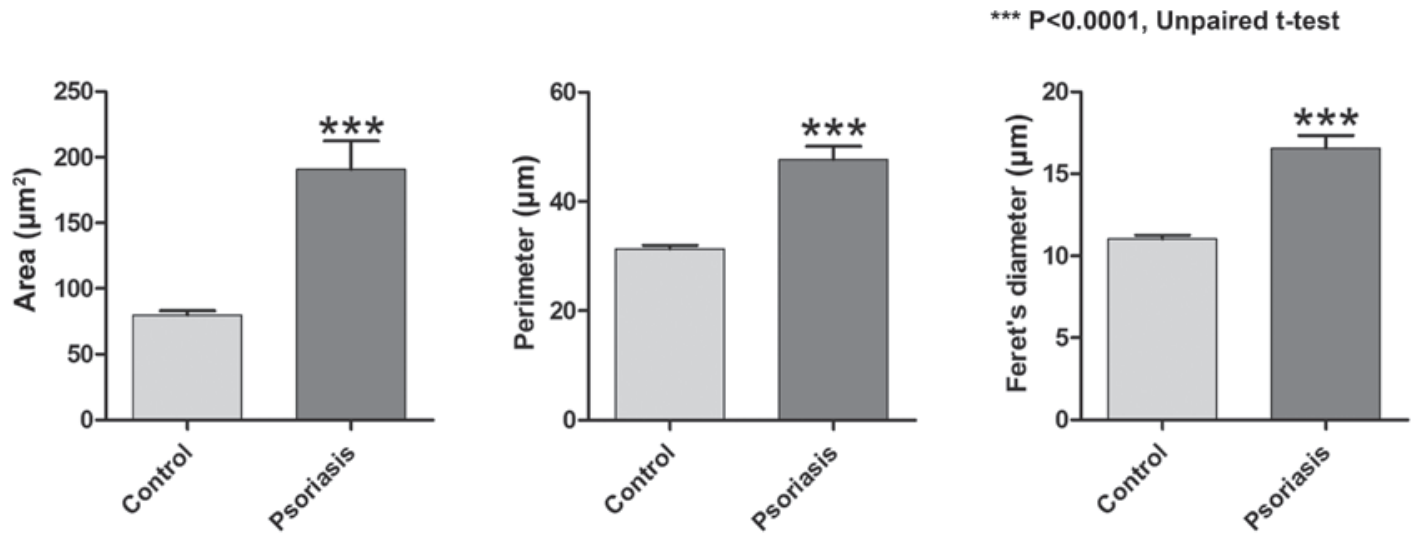

Figure 6. Comparison of parameters of dermal capillaries (area, perimeter, Feret's diameter) between the control group and the psoriasis group. In the lesional skin, all three parameters had significantly higher values compared with those of healthy skin. Values are expressed as the mean \pm standard deviation. **** $\mathrm{P}<0.0001$

dermal capillaries, the mean value in the Psoriasis group was significantly higher than that in the Control group $(190.8 \pm 21.65$ vs. $79.94 \pm 3.122 \mu \mathrm{m} ; \mathrm{P}<0.0001)$. The perimeter of dermal capillaries was also increased in the lesional skin compared that in the healthy skin $(47.67 \pm 2.410$ vs. $31.35 \pm 0.621 \mu \mathrm{m} ; \mathrm{P}<0.0001)$. The Feret's diameter of dermal capillaries had a mean value of $16.55 \pm 0.788 \mu \mathrm{m}$ in the skin affected by psoriasis compared with $11.03 \pm 0.217 \mu \mathrm{m}$ in the skin of healthy subjects, and the difference was statistically significant $(\mathrm{P}<0.0001$; Fig. 6).

\section{Discussion}

The present study used RCM to successfully assess micromorphological changes of cutaneous tissue in psoriasis vulgaris lesions. The results are in line with those of previous studies indicating that the RCM technique may be employed to easily identify characteristic histological features of psoriasis in a noninvasive and reproducible manner (9-12,19-24).

Furthermore, in vivo RCM proved to be useful in differentiating plaque psoriasis from other erythematosquamous skin diseases $(25,26)$ and to be able to distinguish stable from unstable psoriasis plaques $(27,28)$.

The present study focused on the evaluation of skin vessels and dermal papillae and proposes a novel method for the objective assessment of plaque psoriasis lesions using the in vivo RCM technique, which is potentially applicable in clinical studies and in monitoring treatment responses.

Of note, the horizontal sections at the dermo-epidermal junction exhibited an increased percentage of papillary dermal tissue relative to the total examined cutaneous tissue area. Furthermore, it was demonstrated that RCM was capable of determining parameters of dermal papillae's (area, perimeter and Feret's diameter), whose mean values were significantly increased compared with those of the mean values in healthy skin.

A series of previous studies evaluated micromorphological parameters by RCM to assess the dynamic changes occurring in lesions of psoriasis during various treatments (20,29-32), emphasizing the role of subclinical psoriatic skin vessels and dermal papillae in the monitoring of the effectiveness of psoriasis therapy and demonstrating an improved predictability of recurrence following treatment compared with the
PASI clinical score (29). In addition to using parameters of the dermal capillaries and dermal papillae (section area, perimeter and Feret's diameter), the present study included the percentage of papillary dermal tissue per total examined skin and the number of capillary vessels per lesional dermal papilla as novel parameters for a more thorough evaluation of psoriasis lesions. Tracking of histological parameters during therapy provides valuable information on the therapeutic response, the mechanism of action of therapeutic agents and the pathological mechanisms involved in the occurrence of the cutaneous lesions. The method suggested by the present study may be utilized to assess these parameters in an objective manner.

Considering that the dermal microvasculature is affected in a broad spectrum of dermatological disorders, the possibility of studying microvascular changes may be helpful in understanding the underlying pathophysiological mechanisms. In vivo RCM has the advantage of real-time examination of blood flow and dynamic evaluation of morphological changes, while preserving the integrity of the skin. In addition, the cellular resolution of in vivo RCM allows for evaluation of neutrophil migration and accumulation in active psoriatic lesions $(33,34)$. Neutrophils have an established role in the pathophysiology of psoriasis and pathognomonic collections of neutrophils are frequently observed as clusters of highly refractile round to polygonal cells at the level of the stratum spinosum and stratum corneum, where they form micropustules of Kogoj and microabscesses of Munro, respectively $(22,33,34)$.

In vivo confocal microscopy also allows for assessing dermal reactions at the microvascular level induced by exposure to ultraviolet light (35), smoking (36), topical capsaicin (16) or temperature changes $(37,38)$. In addition, this imaging technique was successfully used to verify the viability of skin grafts (39) and musculocutaneous flaps (40).

Dilated and tortuous dermal capillaries represent a histological feature of psoriasis vulgaris $(11,12,41)$, which has been successfully evaluated by RCM. Vessels that run horizontally and perpendicularly to the surface are seen as canalicular or round dark spaces. It has been observed that, compared with the skin of healthy subjects, capillaries examined in lesions of psoriasis have higher mean values of micromorphological 
parameters (area, perimeter and Feret's diameter) and that for each papilla, the number of capillary sections is higher. It should be noted that in the pathogenesis of psoriasis, dermal capillaries become tortuous, describing multiple loops in their path $(11,12,41,42)$, so the horizontal sections made by RCM examination are likely to display several sections of the same capillary vessel.

In the context of major challenges for healthcare systems and the pharmaceutical industry (43), it is currently of interest to use non-invasive tools for the diagnosis of the skin pathologies, which are more readily accepted by the patient and preserve the integrity of examined skin. In the future, RCM may serve as a method for non-invasive and definitive diagnosis of psoriasis vulgaris, for monitoring the evolution of the lesions at the micromorphological level under various treatments and for better understanding the pathophysiological processes that occur in the evolution of this disease. Until then, further study is required in order to establish the precise diagnostic criteria that may be applied in everyday practice.

\section{Acknowledgements}

The present study was partly supported by grants from the Executive Agency for Higher Education, Research, Development and Innovation (grant no. PNII-PT-PCCA-2013-4-1386; project 185/2014) and by the Young Researchers Grant (no. 33891/2014) of Carol Davila University of Medicine and Pharmacy (Bucharest, Romania).

\section{References}

1. Naldi L: Epidemiology of psoriasis. Curr Drug Targets Inflamm Allergy 3: 121-128, 2004

2. Parisi R, Symmons DP, Griffiths CE and Ashcroft DM Identification and Management of Psoriasis and Associated ComorbidiTy (IMPACT) project team: Global epidemiology of psoriasis: A systematic review of incidence and prevalence. J Invest Dermatol 133: 377-385, 2013.

3. Caruntu C, Grigore C, Caruntu A, Diaconeasa A and Boda D: The role of stress in skin diseases. Med Interna (Internal Med) 8: 73-84, 2011.

4. Villarreal-Martínez A, Gallardo-Blanco H, Cerda-Flores R, Torres-Muñoz I, Gómez-Flores M, Salas-Alanís J, OcampoCandiani J and Martínez-Garza L: Candidate gene polymorphisms and risk of psoriasis: A pilot study. Exp Ther Med 11: $1217-1222,2016$

5. Chen W, Xie K, Liu X and Chen H: Identification of key pathways and genes in psoriasis via gene microarray analysis. Mol Med Rep 13: 2327-2337, 2016.

6. Boda D, Negrei C, Nicolescu F and Bălălău C: Assessment of some oxidative stress parameters in methotrexate treated psoriasis patients. Farmacia 62: 704-710, 2014.

7. Negrei C, Arsene AL, Toderescu CD, Boda D and Ilie M: Acitretin treatment in psoriasis may influence the cell membrane fluidity. Farmacia 60: 767-771, 2012.

8. Schön MP and Boehncke WH: Psoriasis. N Engl J Med 352: $1899-1912,2005$

9. Wolberink EA, van Erp PE, Teussink MM, van de Kerkhof PC and Gerritsen MJ: Cellular features of psoriatic skin: Imaging and quantification using in vivo reflectance confocal microscopy. Cytometry B Clin Cytom 80: 141-149, 2011.

10. Wolberink EA, van Erp PE, de Boer-van Huizen RT, van de KerkhofPC and Gerritsen MJ: Reflectance confocalmicroscopy: An effective tool for monitoring ultraviolet B phototherapy in psoriasis. Br J Dermatol 167: 396-403, 2012.

11. Căruntu C, Boda D, Căruntu A, Rotaru M, Baderca F and Zurac S: In vivo imaging techniques for psoriatic lesions. Rom J Morphol Embryol 55 (3 Suppl): S1191-S1196, 2014.
12. Caruntu C, Boda D, Dumitrascu G, Constantin C and Neagu M: Proteomics focusing on immune markers in psoriatic arthritis. Biomark Med 9: 513-528, 2015.

13. Diaconeasa A, Boda D, Neagu M, Constantin C, Căruntu C, Vlădău L and Guţu D: The role of confocal microscopy in the dermato-oncology practice. J Med Life 4: 63-74, 2011.

14. Căruntu C, Boda D, Guţu DE and Căruntu A: In vivo reflectance confocal microscopy of basal cell carcinoma with cystic degeneration. Rom J Morphol Embryol 55: 1437-1441, 2014.

15. Ghita MA, Caruntu C, Rosca AE, Kaleshi H, Caruntu A Moraru L, Docea AO, Zurac S, Boda D, Neagu M, et al: Reflectance confocal microscopy and dermoscopy for in vivo, non-invasive skin imaging of superficial basal cell carcinoma. Oncol Lett 11: 3019-3024, 2016.

16. Căruntu C and Boda D: Evaluation through in vivo reflectance confocal microscopy of the cutaneous neurogenic inflammatory reaction induced by capsaicin in human subjects. J Biomed Opt 17: 085003, 2012.

17. Neagu M, Caruntu C, Constantin C, Boda D, Zurac S, Spandidos DA and Tsatsakis AM: Chemically induced skin carcinogenesis: Updates in experimental models (Review). Oncol Rep 35: 2516-2528, 2016.

18. Rajadhyaksha M, Grossman M, Esterowitz D, Webb RH and Anderson RR: In vivo confocal scanning laser microscopy of human skin: Melanin provides strong contrast. J Invest Dermatol 104: 946-952, 1995.

19. González S, Rajadhyaksha M, Rubinstein G and Anderson RR: Characterization of psoriasis in vivo by reflectance confocal microscopy. J Med 30: 337-356, 1999.

20. Ardigò M, Agozzino M, Longo C, Conti A, Di Lernia V, Berardesca E and Pellacani G: Psoriasis plaque test with confocal microscopy: Evaluation of different microscopic response pathways in NSAID and steroid treated lesions. Skin Res Technol 19: 417-423, 2013.

21. Archid R, Patzelt A, Lange-Asschenfeldt B, Ahmad SS, Ulrich M, Stockfleth E, Philipp S, Sterry W and Lademann J: Confocal laser-scanning microscopy of capillaries in normal and psoriatic skin. J Biomed Opt 17: 101511, 2012.

22. Zhong LS, Wei ZP and Liu YQ: Sensitivity and specificity of Munro microabscess detected by reflectance confocal microscopy in the diagnosis of psoriasis vulgaris. J Dermatol 39: 282-283, 2012.

23. Agozzino M, Gonzalez S and Ardigò M: Reflectance confocal microscopy for inflammatory skin diseases. Actas Dermosifiliogr 107: 631-639, 2016.

24. Ardigo M, Cota C, Berardesca E and González S: Concordance between in vivo reflectance confocal microscopy and histology in the evaluation of plaque psoriasis. J Eur Acad Dermatol Venereol 23: 660-667,2009.

25. Agozzino M, Berardesca E, Donadio C, Franceschini C, de Felice CM, Cavallotti C, Sperduti I and Ardigò M: Reflectance confocal microscopy features of seborrheic dermatitis for plaque psoriasis differentiation. Dermatology 229: 215-221, 2014.

26. Koller S, Gerger A, Ahlgrimm-Siess V, Weger W, Smolle J and Hofmann-Wellenhof R: In vivo reflectance confocal microscopy of erythematosquamous skin diseases. Exp Dermatol 18: 536-540, 2009.

27. Hoogedoorn L, Gerritsen MJ, Wolberink EA, Peppelman M, van de Kerkhof PC and van Erp PE: A four-phase strategy for the implementation of reflectance confocal microscopy in dermatology. J Eur Acad Dermatol Venereol 30: 1308-1314, 2016.

28. Hoogedoorn L, Wolberink EA, van de Kerkhof PC, Hendriks JC, Gerritsen MJ and van Erp PE: Noninvasive differentiation between stable and unstable chronic plaque psoriasis using in vivo reflectance confocal microscopy. J Am Acad Dermatol 73: 870-872, 2015.

29. Archid R, Duerr HP, Patzelt A, Philipp S, Röwert-Huber HJ, Ulrich M, Meinke MC, Knorr F and Lademann J: Relationship between histological and clinical course of psoriasis: A pilot investigation by reflectance confocal microscopy during goeckerman treatment. Skin Pharmacol Physiol 29: 47-54, 2016.

30. Başaran YK, Gürel MS, Erdemir AT, Turan E, Yurt N and Bağci IS: Evaluation of the response to treatment of psoriasis vulgaris with reflectance confocal microscopy. Skin Res Technol 21: 18-24, 2015.

31. Lacarrubba F, Pellacani G, Gurgone S, Verzì AE and Micali G: Advances in non-invasive techniques as aids to the diagnosis and monitoring of therapeutic response in plaque psoriasis: A review. Int J Dermatol 54: 626-634, 2015. 
32. Ardigò M, Agozzino M, Longo C, Lallas A, Di Lernia V, Fabiano A, Conti A, Sperduti I, Argenziano G, Berardesca E and Pellacani G: Reflectance confocal microscopy for plaque psoriasis therapeutic follow-up during an anti-TNF- $\alpha$ monoclonal antibody: An observational multicenter study. J Eur Acad Dermatol Venereol 29: 2363-2368, 2015.

33. Wolberink EA, Peppelman M, van de Kerkhof PC, van Erp PE and Gerritsen MJ: Establishing the dynamics of neutrophil accumulation in vivo by reflectance confocal microscopy. Exp Dermatol 23: 184-188, 2014.

34. Moscarella E, Argenziano G, Lallas A, Pellacani G and Longo C: Confocal microscopy: A new era in understanding the pathophysiologic background of inflammatory skin diseases. Exp Dermatol 23: 320-321, 2014.

35. Yamashita T, Akita H, Astner S, Miyakawa M, Lerner EA and González S: In vivo assessment of pigmentary and vascular compartments changes in UVA exposed skin by reflectance-mode confocal microscopy. Exp Dermatol 16: 905-911, 2007.

36. Altintas MA, Altintas AA, Guggenheim M, Gohritz A, Meyer-Marcotty Mand VogtPM: Reflectance confocal-laser-scanning microscopy in vivo assessments of cigarette-induced dynamic alterations of cutaneous microcirculation on histomorphological level. Microsc Res Tech 72: 347-350, 2009.

37. Altintas MA, Meyer-Marcotty M, Altintas AA, Guggenheim M, Gohritz A, Aust MC and Vogt PM: In vivo reflectance-mode confocal microscopy provides insights in human skin microcirculation and histomorphology. Comput Med Imaging Graph 33: 532-536, 2009.

38. Altintas MA, Altintas AA, Guggenheim M, Steiert AE, Aust MC, Niederbichler AD, Herold C and Vogt PM: Insight in human skin microcirculation using in vivo reflectance-mode confocal laser scanning microscopy. J Digit Imaging 23: 475-481, 2010.
39. Greenwood J, Amjadi M, Dearman B and Mackie I: Real-time demonstration of split skin graft inosculation and integra dermal matrix neovascularization using confocal laser scanning microscopy. Eplasty 9: e33, 2009.

40. Altintas MA, Altintas AA, Guggenheim M, Knobloch K, Niederbichler AD and Vogt PM: Monitoring of microcirculation in free transferred musculocutaneous latissimus dorsi flaps by confocal laser scanning microscopy-a promising non-invasive methodical approach. J Plast Reconstr Aesthet Surg 63: 111-117, 2010.

41. Toussaint $\mathrm{S}$ and Kamino H: Noninfectious erythematous, papular and squamous diseases. In: Lever's Histopathology of the Skin. Elder D, Elenitsas R, Jaworsky C and Johnson B Jr (eds). 8th edition. Lippincott-Raven, Philadelphia, PA, pp151-184, 1997.

42. Tudoraşcu I, Sfredel V, Riza AL, Dănciulescu Miulescu R, Ianoşi SL and Dănoiu S: Motor unit changes in normal ageing: A brief review. Rom J Morphol Embryol 55: 1295-1301, 2014.

43. Raţiu MP, Purcărea I, Popa F, Purcărea VL, Purcărea TV, Lupuleasa D and Boda D: Escaping the economic turn down through performing employees, creative leaders and growth driver capabilities in the Romanian pharmaceutical industry. Farmacia 59: 119-130, 2011.

This work is licensed under a Creative Commons Attribution-NonCommercial-NoDerivatives 4.0 International (CC BY-NC-ND 4.0) License. 